\title{
Simulation of the Impact on the Workload of the Enlargement of the Clinical Staff of a Specialistic Reference Center
}

\author{
Noemi GIORDANO ${ }^{\mathrm{a}, 1}$, Samanta ROSATI ${ }^{\mathrm{a}}$, Federica VALERI ${ }^{\mathrm{b}}$, Alessandra \\ BORCHIELLINI $^{\mathrm{b}}$ and Gabriella BALESTRA ${ }^{\mathrm{a}}$ \\ a Department of Electronics and Telecommunications, Politecnico di Torino, Italy \\ ${ }^{\mathrm{b}}$ Oncologic Department, Città della Salute e della Scienza di Torino, Torino, Italy
}

\begin{abstract}
Quality of care and patient satisfaction are important aspects of high standard care. If clinical staff is subject to an elevated workload there is a possible decrease of both. This justifies the development of tools to quantify the workload and to find organizational changes that will normalize it. We have previously developed a simulation system to quantify the workload of the staff working in a regional reference center for the treatment of bleeding and hemorrhagic disorders. The goal of this new work is to simulate, through an agent-based model, the impact of adding a physician to the staff. Ten sets of initial parameters were defined to simulate ten typical weeks. Results show that the introduction of the new physician together with a second ambulatory room can reduce the workload of all the staff to the expected 8-hour. In this situation, in which the staff workload does not exceed the daily capacity, we may suppose that an increase in the quality of care and patient satisfaction will be possible.
\end{abstract}

Keywords. ABM, simulation, process modelling, clinical process, NetLogo

\section{Introduction}

The effects of an exaggerated workload for physicians and clinical staff have been widely explored in literature. The quality of care was proved to be negatively impacted by high workloads in terms of clinical outcomes, patient safety and mortality (1-3), but also the patient-physician interaction and the consequent patient satisfaction $(3,4)$ strongly suffer from high workload. Hence, the quantification of the physicians' workload, along with strategies for reducing it, are issues worth of being explored.

Nevertheless, strategies for reducing the workload of clinical staff typically involve organizational changes which may be difficult to implement for economic or logistical reasons. In this context, simulations represent a valuable tool to evaluate the effect of the changes before putting them in place $(5,6)$.

In a previous work (7), we have designed and tested an Agent-based model of the activities of a reference center for the treatment of hemorrhagic and bleeding disorders located at the "Città della Salute e della Scienza" hospital in Turin, Italy. The center staff consists of two specialized physicians whose average workload accounts for over

${ }^{1}$ Corresponding Author: Noemi Giordano, Department of Electronics and Telecommunications, Politecnico di Torino, Corso Duca degli Abruzzi 24, 10129 Torino, Italy; E-mail: noemi.giordano@polito.it. 
10.2 hours a day, as quantified through on-field studies and data collection (8). In particular, from the on-field data collection, we found that the bottleneck of the daily organization of the activities of the physicians is the ambulatory care. Thus, we have simulated the impact of increasing the number of ambulatory rooms, using the staff currently available in the center, but found that it was not sufficient to reduce the daily physicians' workload to the expected 8 hours (7).

The goal of this novel work is to simulate the effect of adding both a new ambulatory room and a third physician to the center staff. In particular, we aim at quantifying if these two conditions together can reduce the physicians' workload down to a regular 8-hour shift and at investigating its further potential positive effects to the logistics of the center.

\section{Materials and Methods}

\subsection{ABM model}

The Agent-based model (ABM) we used for the study was developed and implemented using NetLogo 6.1.0 platform (9) for its simplicity in use, scalability and computational modelling strength $(10,11)$.

We defined three types of agents (physician, outpatient and inpatient) who move and act in a simulation environment defined accordingly to the scaled map of the real hospital. We modelled 7 processes (Ambulatory care; Consultations, required from both other departments and other hospitals; Administrative procedures; Therapeutic plans; Clinical protocols; Interruptions during ambulatory care, mainly due to requests of phone advice; Other activities, such as research and teaching) and defined the model parameters statistically using previously collected data.

\subsection{Dimensioning of the workload of the additional physician}

The simulation developed in Netlogo modeled activities included in all processes previously mentioned. The activities were scheduled during the five working days differently for each physician and we call this schedule agenda.

The agenda of the two physicians currently enrolled in the center was organized taking into account their weekly organization of the activities. For the new physician, we defined a daily agenda coherent to that of the other two physicians, but we charged $\mathrm{him} /$ her only with the three core activities of the center: ambulatory care, internal advice and management of the therapeutic plan. Consequently, only these three processes were simulated for the third physician.

Since ambulatory care is the main cause of the higher workload, we dimensioned the worktime of the third physician with the scope of reducing the number of ambulatory patients assigned to each physician to the maximum allowed by the 4-hour time slot.

We quantified the average number of exceeding patients day-by-day for each physician. Then, we assigned the weekly average exceeding patients to the third physician and computed the corresponding number of ambulatory care slots needed to manage these patients. We then defined the agenda of the third physician day-by-day, considering two ambulatory rooms available for the center, to be used simultaneously.

Concerning internal advice and management of the therapeutic plans, we divided the daily number of patients equally over the three physicians. 


\subsection{Design of the simulations}

The changes described in the previous section were implemented starting from the simulation model described in (7) and adding a new agent of type "physician". In the simulation environment, this new agent performed the activities according to his scheduled agenda. No modifications to the model were made concerning either the model rules or the statistical definition of the simulation parameters. This choice was dictated by the will of maintaining the context coherent with the actual center organization.

We simulated the impact of the introduction of a third physician to the center staff over ten simulated weeks. The period of a week was chosen because most activities of the center are planned and scheduled on a weekly basis. The weeks were simulated by randomly defining 10 sets of initial parameters.

Validation was performed comparing the simulation results with the data previously collected on field. The results of the three simulations were statistically compared using a one-way ANOVA $(\alpha=0.05)$ followed by a post hoc Bonferroni test.

\section{Results and Discussion}

\subsection{Model Validation}

Figure 1 shows the validation results. In particular, the partition of the average time daily dedicated to each process is presented, as obtained respectively in a) the collected data and $b$ ) the results of the simulations.

a) Collected Data

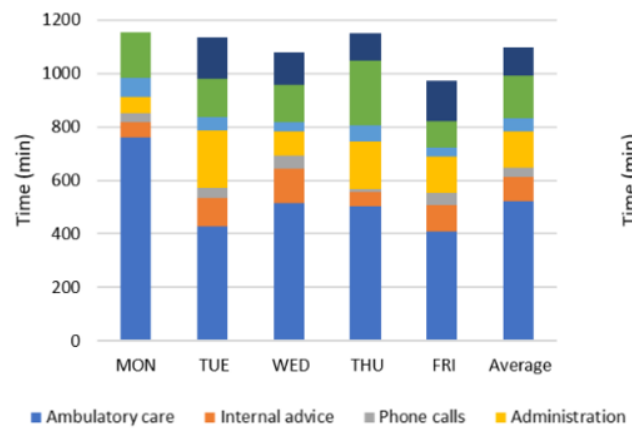

b) Results of the simulation

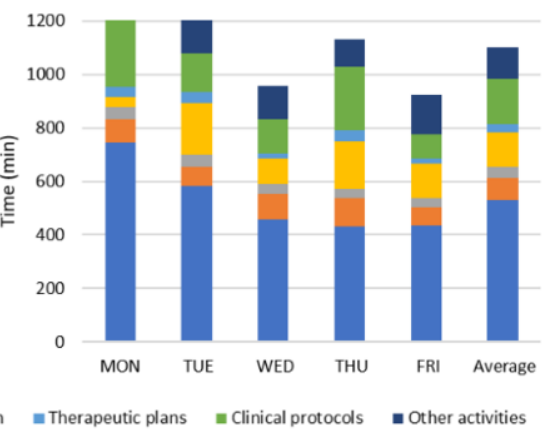

Figure 1. Comparison of the time dedicated to each process in the collected data (a) and the results of the simulations (b).

Data shown in Figure 1 refer to the overall time dedicated to each process, i.e. the sum of the time dedicated by each physician. In other words, it represents the overall center workload during an average week. The results show that the model is capable of accurately reproducing the center average workload, both in its absolute value and in terms of partition of the activities. 


\subsection{Results of the simulations}

Figure 2 presents the results of the new scenario (green bars) compared to the actual situation (blue bars) and the scenario where only an additional ambulatory room was added (yellow bars). The comparison was performed day-by-day and in average over the five weekdays. Values are reported in terms of mean and standard deviation over the 10 simulations.

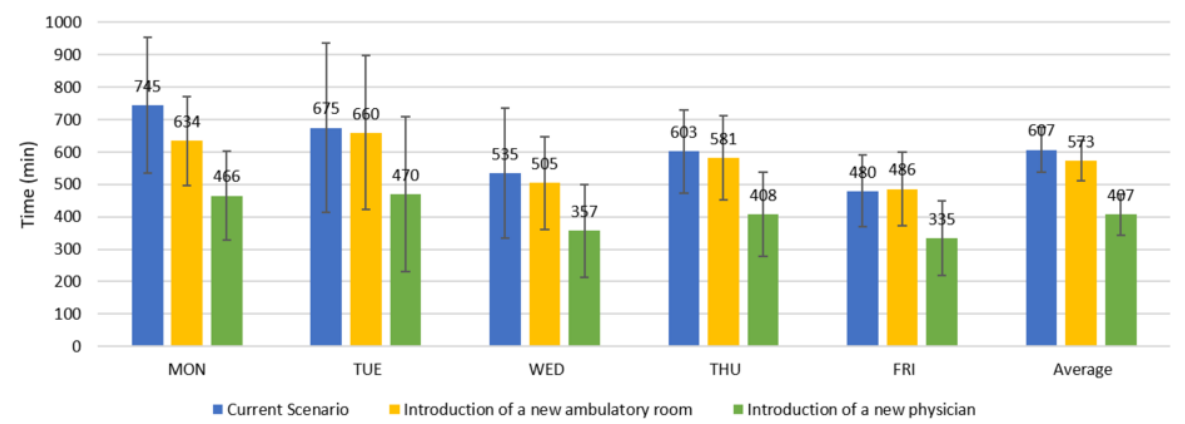

Figure 2. Simulated daily workload, averaged over the physicians, in the three scenarios (current scenario, introduction of a secondary ambulatory room, introduction of a new physician together with a second ambulatory room). Bars and whiskers represent the mean and the standard deviation over 10 simulations, respectively.

The results presented above confirm that the introduction of a third physician leads to a drastic reduction of the average daily workload. This is also supported by the results of the ANOVA $(\mathrm{p}<0.001)$. In particular, the post hoc Bonferroni test shows that only the last scenario (new physician with second ambulatory room) is statistically different from the other two.

To have a deeper insight into the effects of the organizational change to the workload of physicians, the daily workload divided per physician is presented in Figure 3 (mean and standard deviation over the 10 simulations).

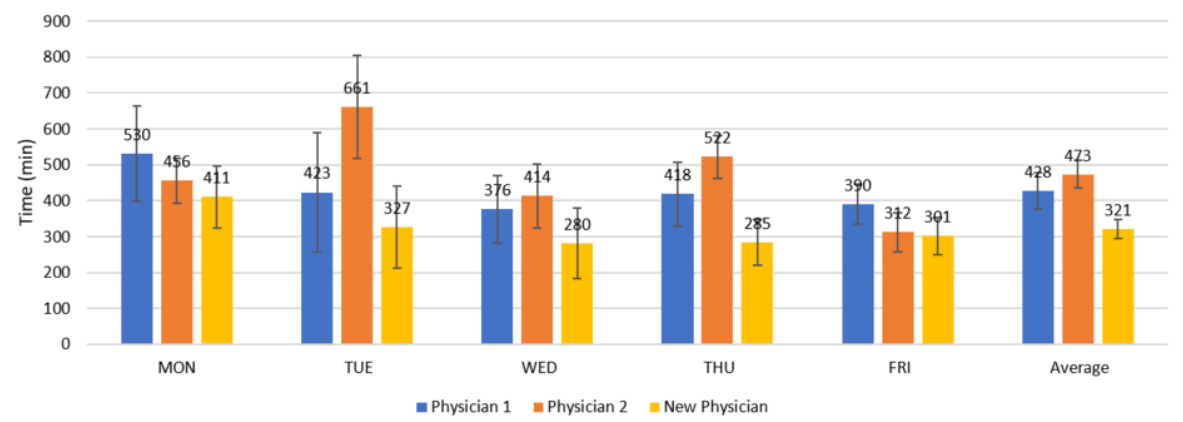

Figure 3. Simulated daily average workload for each physician after the introduction of the new physician together with a second ambulatory room (bars and whiskers represent the mean and the standard deviation over 10 simulations, respectively). 
The daily workload is not equally distributed over the weekdays because the physician agenda was not optimized. The average results show that, with some further changes in the weekly scheduling, normal shifts can be achieved and that there is the possibility to increasing the quality of care for example reducing the waiting lists for ambulatory visit or having more time to participate in clinical trials.

\section{Conclusions}

This work presents the last part of a project aimed first to quantify the workload of center actual staff and then to simulate the impact of different scenarios in reducing the workload of the staff. In a previous work (8), we presented the process modeling and the data collected during ten weeks that gave evidence of an average workload definitely higher than normal. The results of our simulations show that with two ambulatory rooms and another physician the average daily workload of each physician is reduced from 10 hours to normal. In this situation, in which the staff workload does not exceed the daily capacity, we may suppose that an increase in the quality of care and patient satisfaction will be possible.

\section{References}

[1] Kotze K, van der Westhuizen HM, van Loggerenberg E, Jawitz F, Ehrlich R. Doctors' extended shifts as risk to practitioner and patient: South Africa as a case study. Int J Environ Res Public Health. 2020;17(16):1-10.

[2] van den Hombergh P, Künzi B, Elwyn G, van Doremalen J, Akkermans R, Grol R, et al. High workload and job stress are associated with lower practice performance in general practice: an observational study in 239 general practices in the Netherlands. BMC Health Serv Res. 2009 Dec;9(1):118.

[3] Williams ES, Rondeau K V., Xiao Q, Francescutti LH. Heavy physician workloads: Impact on physician attitudes and outcomes. Heal Serv Manag Res. 2007;20(4):261-9.

[4] Garcia-Lacalle J. Balancing physician workload and patient satisfaction: A dilemma for hospital managers. Public Money Manag. 2010;30(3):183-8.

[5] Fone D, Hollinghurst S, Temple M, Round A, Lester N, Weightman A, et al. Systematic review of the use and value of computer simulation modelling in population health and health care delivery. J Public Health (Bangkok). 2003;25(4):325-35.

[6] Denton BT. Handbook of Healthcare Operations Management. New York, New York: Springer; 2013; p. $45-74$.

[7] Giordano N, Rosati S, Valeri F, Borchiellini A, Balestra G. Agent-Based Modeling and Simulation of Care Delivery for Patients with Thrombotic and Bleeding Disorders. In: Studies in health technology and informatics Proceedings of MIE 2020, 2020; p. 1193-4.

[8] Rosati S, Valeri F, Borchiellini A, Gianfreda CM, Balestra G. Characterization of Physicians Workload in a Reference Center for the Treatment of Thrombotic and Bleeding Disorders *. In: Proceedings of the 2019 40th Annual International Conference of the IEEE Engineering in Medicine and Biology Society (EMBC)40th Annual International Conference of the IEEE Engineering in Medicine and Biology Society (EMBC). IEEE; 2019; p.1359-62.

[9] Wilensky U. NetLogo. Center for Connected Learning and Computer-Based Modeling Northwestern University Evanston. 1999. Available at: https://ccl.northwestern.edu/netlogo/

[10] Abar S, Theodoropoulos GK, Lemarinier P, Hare GMPO. Agent Based Modelling and Simulation tools: A review of the state-of-art software. Comput Sci Rev.. 2017;24:13-33.

[11] Wilensky U, Rand W. An Introduction to Agent-Based Modeling. Cambridge, Massachusetts: The MIT Press; 2015; p. 1-43. 